\title{
The roles of glycosphingolipids in the proliferation and neural differentiation of mouse embryonic stem cells
}

\author{
Ji-Ung Jung ${ }^{1 *}$, Kinarm $\mathrm{Ko}^{2 *}$, Dae-Hoon Lee ${ }^{3}$, \\ Kisung Ko ${ }^{1}$, Kyu-Tae Chang ${ }^{4,5}$ and Young-Kug Choo ${ }^{1,5}$ \\ ${ }^{1}$ Department of Biological Science \\ College of Natural Sciences \\ Wonkwang University \\ Iksan 570-749, Korea \\ ${ }^{2}$ Department of Cell and Developmental Biology \\ Max Planck Institute for Molecular Biomedicine \\ Muenster 48149, Germany \\ ${ }^{3}$ The Institute of Molecular Medicine and Genomics and \\ Department of Neurology \\ Medical College of Georgia \\ Augusta, GA 30912, USA \\ ${ }^{4}$ National Primate Research Center (NPRC) \\ Korea Research Institute of Bioscience and Biotechnology (KRIBB), \\ Ochang 363-883, Korea \\ ${ }^{5}$ Corresponding authors: Tel, 82-63-850-6087; Fax, 82-63-857-8837; \\ E-mail, ykchoo@wku.ac.kr (Y.K. Choo) \\ Tel, 82-43-240-6302; Fax, 82-43-240-6309; \\ E-mail, Changkt@kribb.re.kr (K.T. Chang) \\ *These authors contributed equally to this work. \\ DOI 10.3858/emm.2009.41.12.099
}

Accepted 17 August 2009

Abbreviations: DEAE, diethylaminoethyl; EBs, embryoid bodies; GFAP, glial fibrillary acidic protein; GSLs, glycosphingolipids; HPTLC, high-performance thin-layer chromatography; LIF, leukemia inhibitory factor; MAP-2, microtubule-associated protein 2; MEF, mouse embryonic fibroblast; mESCs, mouse embryonic stem cells; shRNAs, short hairpin RNAs; Ugcg, UDP-glucose:ceramide glucosyltransferase

\footnotetext{
Abstract

Glycosphingolipids including gangliosides play important regulatory roles in cell proliferation and differentiation. UDP-glucose:ceramide glucosyltransferase $(U g c g)$ catalyze the initial step in glycosphingolipids biosynthesis pathway. In this study, Ugcg expression was reduced to approximately $80 \%$ by short hairpin RNAs (shRNAs) to evaluate the roles of glycosphingolipids in proliferation and neural differentiation of mouse embryonic stem cells (mESCs). HPTLC/immunofluorescence analyses of shRNAtransfected mESCs revealed that treatment with Ugcg-shRNA decreased expression of major ganglio-
}

sides, GM3 and GD3. Furthermore, MTT and Western blot/immunofluorescence analyses demonstrated that inhibition of the Ugcg expression in mESCs resulted in decrease of cell proliferation $(P<0.05)$ and decrease of activation of the ERK $1 / 2(P<0.05)$, respectively. To further investigate the role of glycosphingolipids in neural differentiation, the embryoid bodies formed from Ugcg-shRNA transfected mESCs were differentiated into neural cells by treatment with retinoic acid. We found that inhibition of Ugcg expression did not affect embryoid body (EB) differentiation, as judged by morphological comparison and expression of early neural precursor cell marker, nestin, in differentiated EBs. However, RT-PCR/immunofluorescence analyses showed that expression of microtubule-associated protein 2 (MAP-2) for neurons and glial fibrillary acidic protein (GFAP) for glial cells was decreased in neural cells differentiated from the shRNA-transfected mESCs. These results suggest that glycosphingolipids are involved in the proliferation of mESCs through ERK1/2 activation, and that glycosphingolipids play roles in differentiation of neural precursor cells derived from mESCs.

Keywords: cell differentiation; ceramide glucosyltransferase; extracellular signal-regulated MAP kinases; glycosphingolipids; embryonic stem cells; neurons

\section{Introduction}

Glycosphingolipids are primarily found in the outer leaflet of the plasma membrane microdomain on various mammalian cells, and gangliosides are regulated by specific ceramides bound to the Golgi apparatus (Van Meer, 1993). Ceramide, in turn, is modified by the action of glucosylceramide synthase (UDP-glucose:ceramide glucosyltransferase; $U g c g$ ) on the cytosolic surface of the Golgi, which results in the production of glucosylceramide (Figure 1A). Glucosylceramide is then converted to lactosylceramide by the addition of a galactose moiety in the luminal leaflet of the Golgi (Allende and Proia, 2002). Glycosphingolipids are converted to gangliosides by carrying one or more sialic acid residues in the carbohydrate moiety. Successive sialylations of lactosylceramide lead to the formation of monosialoganglioside GM3, disia- 
A

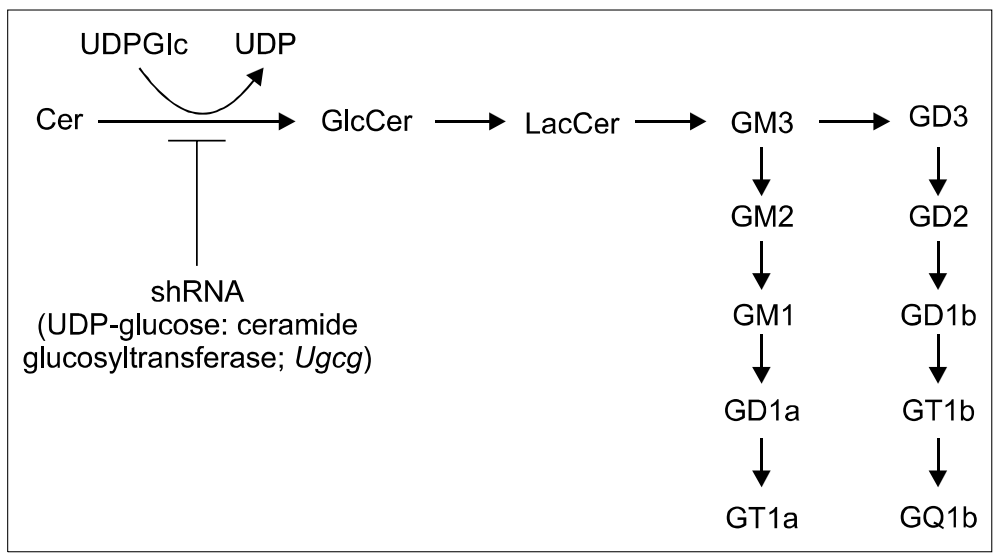

B

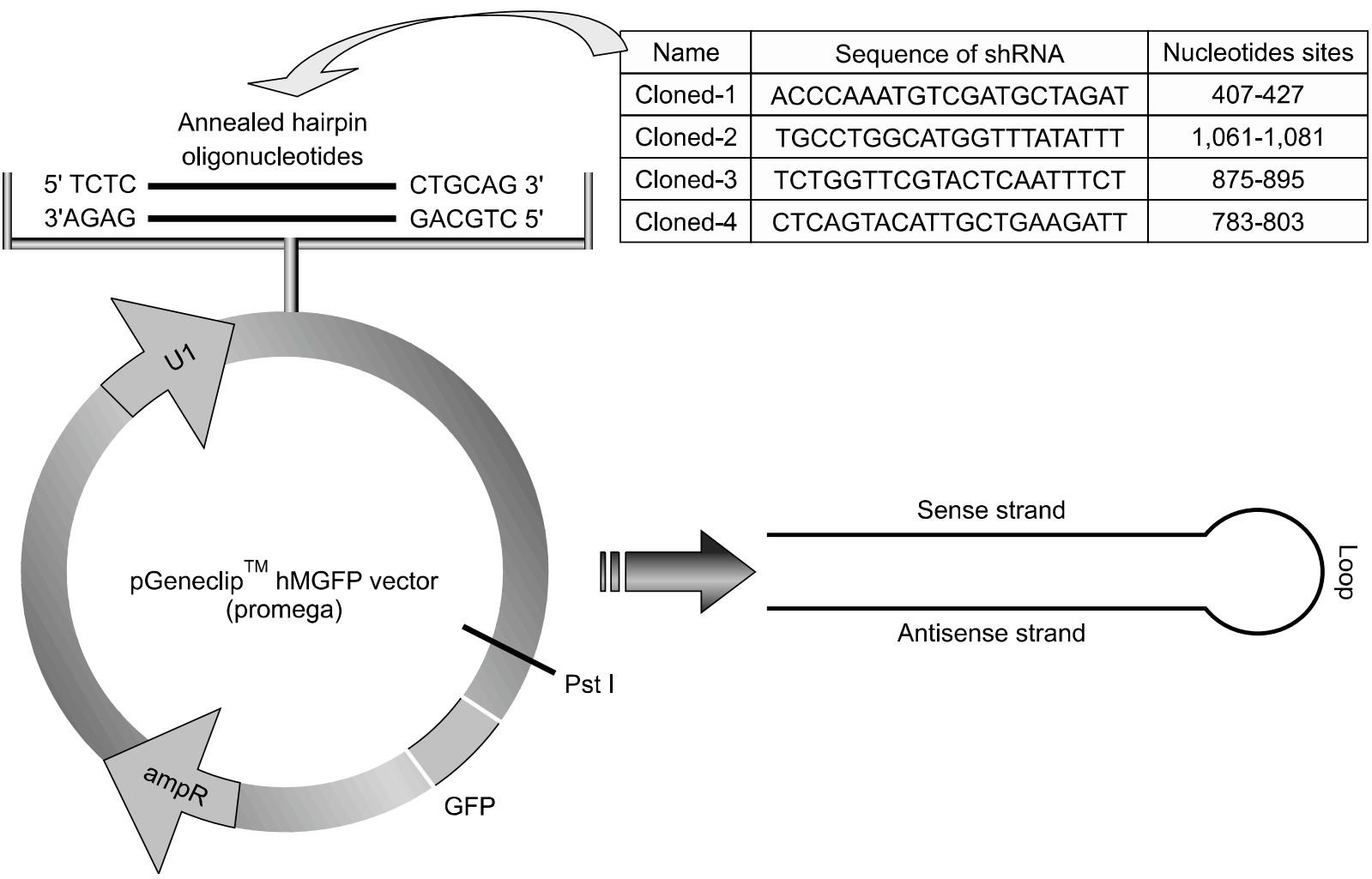

Figure 1. Schematic diagram of the four shRNAs designed to target the Ugcg gene in mESCs. (A) Biosynthetic pathway of glucosylceramide-based glycosphingolipids and gangliosides. Glucosylceramide synthase is the enzyme in the biosynthetic first step for the synthesis of most gangliosides and glycosphingolipids. (B) shRNAs vector. Four shRNAs (cloned 1-4) designed to inhibit expression of the mouse Ugcg gene (UDP-glucose ceramide glucosyltransferase; GenBank. Accession No. NM_011673, SuperArray Bioscience Corp., Frederick, MD).

loganglioside GD3, and trisialoganglioside GT3 (Van Echten and Sandhoff, 1993).

It is known that the biosynthesis and expression of glycosphingolipids are closely correlated with various cellular processes, such as proliferation, differentiation, and apoptosis. Glycosphingolipids, including gangliosides, are known to regulate various signal molecules, such as receptor tyrosine kinases of various growth factor receptors and mitogen-activated protein kinase (MAPK), which are key transducers of extracellular signaling during cell growth and differentiation (Fukumoto et al., 2000; Kimura et al., 2001; Maupas-Schwalm et al., 2004; Stoica et al., 2005; Murozuka et al., 
2007). The composition of glycosphingolipids in cellular membranes is associated with specific cell types and is drastically changed during embryonic development (Yu, 1994; Draper et al., 2002). Glycosphingolipids also appear to be necessary for the central nervous system to function (Kawai et al., 2001) and to be involved in the formation of myelin during neuritogenesis and in neurite outgrowth (Liour et al., 2000). Functions of glycosphingolipids in stem cells were demonstrated by several groups since the first study of gangliosides expression in mouse embryonic stem cells (mESCs) (Kimber et al., 1993). Many approaches have recently been developed to regulate the expression of glycosphingolipids for evaluating their functions. However, the results are various depending on methods and cell lines used in different studies.

Previously, we reported that ganglioside expression was closely related with neural cell specific gene expression in an in vitro model of neural differentiation in $\mathrm{mESCs}$ and that neural differentiation was facilitated by increase of ganglioside expression due to daunorubicin treatment (Lee et al., 2007). However, the chemicals that are used to regulate the biosynthesis of glycosphingolipids have been shown to have side effects, such as cytotoxicity and low specificity, particularly alteration of glycosphingolipids biosynthesis may be actively involved in the acquisition of drug-resistance (Kiura et al., 1998; Prinetti et al., 2006). It has been suggested that a genetic approach for regulating biosynthesis of glycosphingolipids would be appropriated in that the role of glycosphingolipids in the ESCs differentiation process can be directly elucidated. (Yamashita et al., 1999; Liu et al., 2004; Diaz-Font et al., 2006). Therefore, in this study, we investigated the roles of glycosphingolipids in the proliferation and neural differentiation of mESCs by direct inhibition of glycosphingolipid biosynthesis using Ugcg-shRNA.

\section{Results}

\section{Downregulation of the Ugcg mRNA level by shRNAs}

To induce suppression of the Ugcg gene expression, mESCs were transfected with four different Ugcg-targeting shRNAs using a GFP vector containing T7 RNA polymerase under control of the U1 promoter (Figure 1B). We evaluated the alteration of Ugcg mRNA by transfection with Ugcg-shRNAs constructs at $48 \mathrm{~h}$ after transfection. The altered Ugcg gene expression by transfection had no effect on the morphology of the mESCs (Figure 2A). RT-PCR analysis showed that shRNA clone-1 was the most effective in downregulation of $\mathrm{Ugcg}$ gene expression in mESCs (Figure 2B). Therefore, shRNA clone-1 was used in the following experiments.

\section{Downregulation of glycosphingolipids expression by shRNA}

We expected reduction of glycosphingolipids expression in response to Ugcg-shRNA because the biosynthetic pathway of glucosylceramide-based glycosphingolipids begins with glucosylceramide synthase, which is expressed by the Ugcg (Proia, 2003). Therefore, the effect of Ugcg-shRNA on the expression of glycosphingolipids was investigated by HPTLC and immunofluorescence analysis (Figures 2C-2F). HPTLC analysis demonstrated that the glycosphingolipids expression, particularly ganglioside GM3 and GD3, was reduced by $60 \%$ in Ugcg-shRNA transfected cells (Figure 2C, lane 5). Conversely, the expression of gangliosides in mESCs transfected with the negative-shRNA was unchanged (Figure $2 \mathrm{C}$, lane 4). The immunofluorescence assay also showed that the expression levels of GM3 and GD3 in the undifferentiated mESCs decreased in shRNA-transfected $\mathrm{mESCs}$ in comparison to mock-transfected mESCs and control mESCs (Figure 2D, E and F).

\section{Suppression of the $\mathrm{mESC}$ proliferation by inhibition of Ugcg gene expression}

The effect of suppression of $U g c g$ gene expression on the proliferation of mESCs was investigated by MTT assay. mESCs were transfected with negative-shRNA and shRNA-1 for $24 \mathrm{~h}$, and then cell proliferation was measured at $48 \mathrm{~h}$ after transfection. Although the effect was not dramatic, the shRNA-1 significantly reduced $\mathrm{mESC}$ prolifereation by $86 \%$ in comparison to the control cells. Conversely, no significant effect on proliferation was observed in the mock-transfected mESCs when compared to that of the control mESCs $(96 \%)$.

\section{Suppression of MAPK activation by inhibition of Ugcg gene expression}

To determine if suppression of $U g c g$ gene expression inhibited cell proliferation via inhibition of the MAPK pathway (ERK1/2), activation of the MAPK pathway was examined by Western blot in the 10 min serum-stimulated mESCs after $6 \mathrm{~h}$ serum-starvation. The Western blot using a phospho-ERK $1 / 2$ showed that the ERK1/2 was phosphorylated in the cells during maintenance under normal culture conditions (Figure 4A). However, phosphorylation 
A

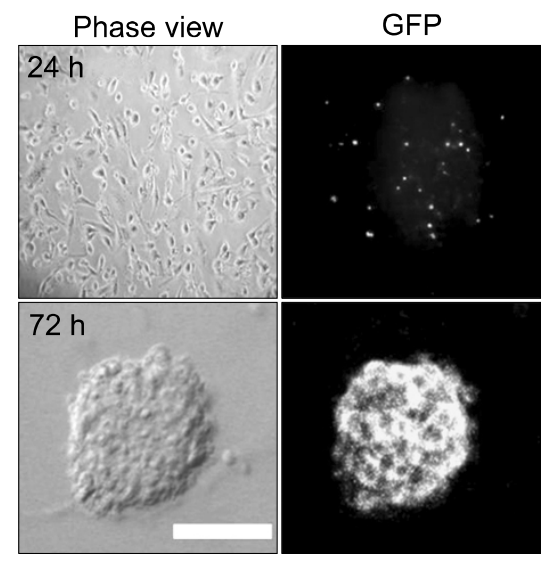

C

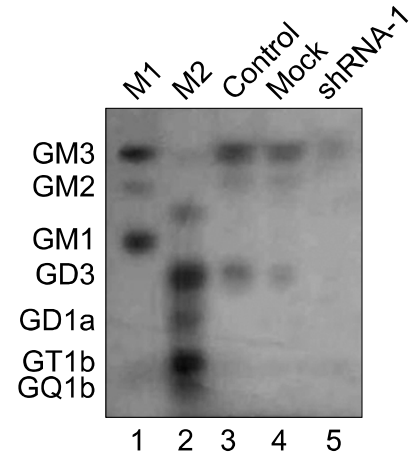

E

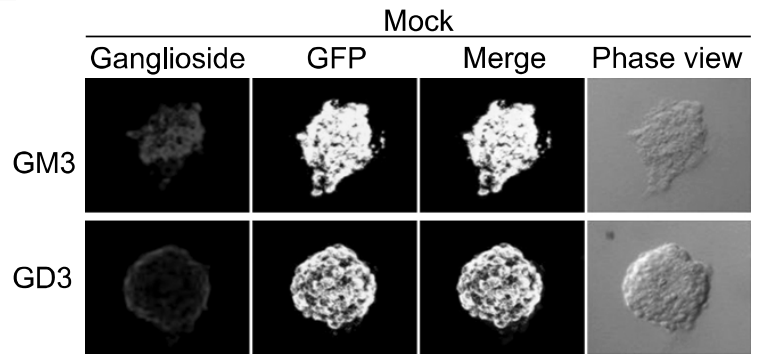

B
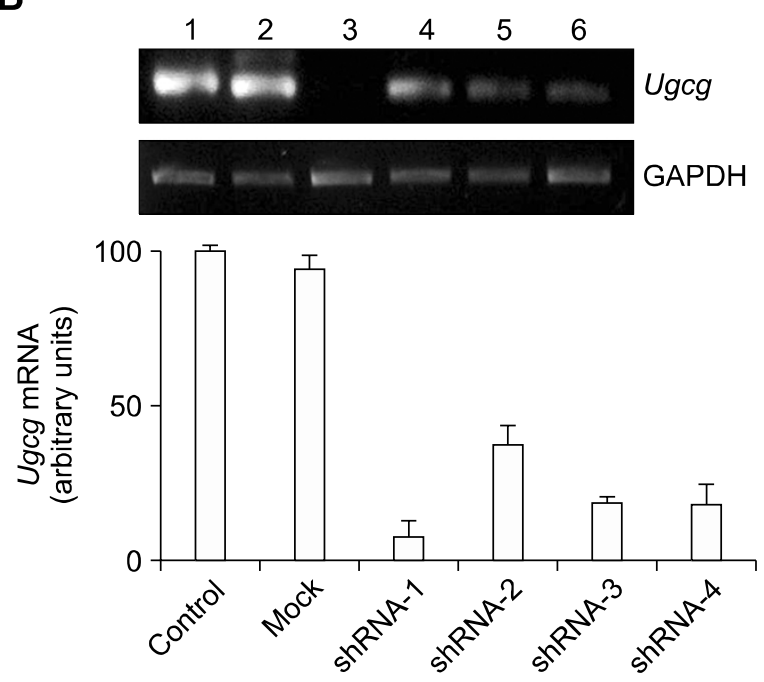

D

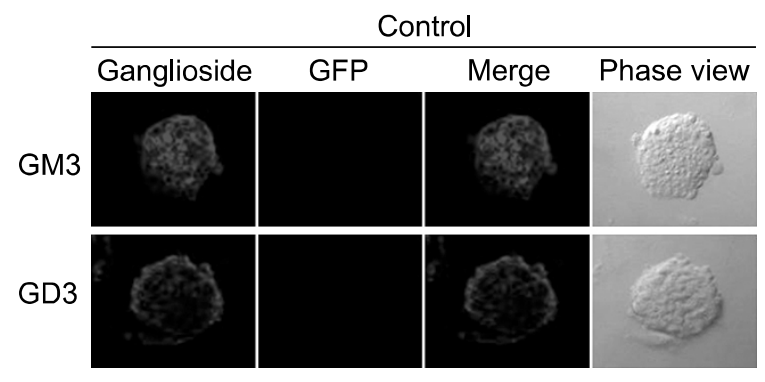

$\mathbf{F}$

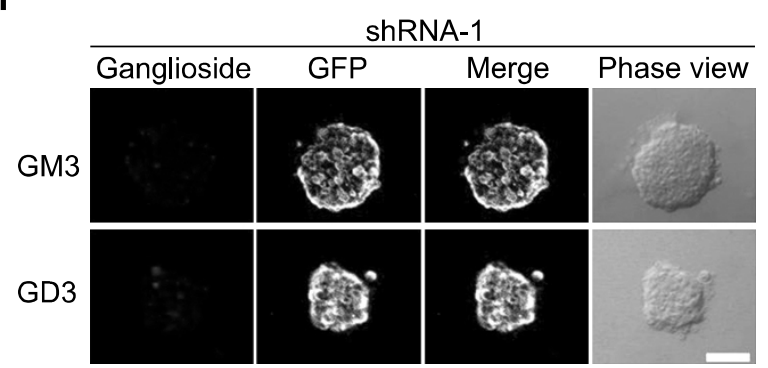

Figure 2. Analysis of Ugcg gene and glycosphingolipids expression on Ugcg shRNAs-transfected mESCs. (A) Transfected mESCs with a normal morphology and GFP fluorescence. $24 \mathrm{~h}, 24 \mathrm{~h}$ after transfection; $72 \mathrm{~h}, 72 \mathrm{~h}$ after transfection (B) RT-PCR analysis of Ugcg gene expression in transfected mESCs. Lane 1, control mESCs; lane 2, mock-transfected mESCs; lane 3, cloned-1 transfected mESCs; lane 4, cloned-2 transfected mESCs; lane 5, cloned-3 transfected mESCs; lane 6, cloned-4 transfected mESCs. RT-PCR was carried out for 35 cycles and expression levels were controlled using GAPDH. (C) Ganglioside extracts were resolved on an HPTLC silica gel plate using chloroform:methanol:0.22\% $\mathrm{CaCl}_{2}(55: 45: 10$, v/v/v), and then visualized with resorcinol-HCl reagent. Lane 1 and 2, ganglioside standard marker; Lane 3, control mESCs; Lane 4, mock-transfected mESCs; Lane 5, shRNA-transfected mESCs. (D-F) The mESCs were subjected to immunofluorescence staining with mAb that was specific for the ganglioside GM3 and GD3. The scale bar represents $100 \mu \mathrm{m}$.

of the ERK1/2 was almost undetectable under serum-free conditions (Figure 4B, lanes 1, 3 and 5). Interestingly, the shRNA-1 transfected mESCs showed inhibition of serum-induced ERK1/2 phos- phorylation $(P<0.05)$ (Figure 4B, lane 6), whereas mock transfection did not affect serum-induced ERK1/2 phosphorylation (Figure 4B, lane 4). In addition, activation of the MAPK was examined 


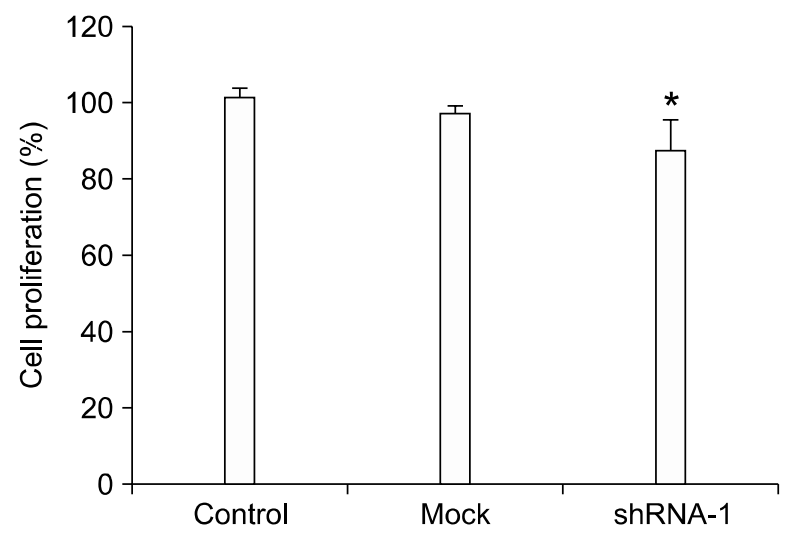

Figure 3. Effect of the suppressed Ugcg gene expression on the proliferation of mESCs. Cells were seeded at a density of $4 \times 10^{4}$ cells/well in 24-well plates and then transfected for $24 \mathrm{~h} .48 \mathrm{~h}$ after transfection, cell proliferation was evaluated by an MTT assay. A low proliferation of mESCs was observed in shRNA-transfected mESCs. The values represent the mean \pm SD of four independent experiments. ${ }^{*} P<0.05$.

using an immunofluorescence analysis (Figure 4C). This analysis showed that phosphorylated ERK1/2 was also expressed in control mESCs and mock-transfected mESCs. However, the intensity of the phosphorylated ERK $1 / 2$ expressed in shRNA-1 transfected mESCs was reduced in comparison to that of mock-transfected mESCs (Figure 4C).

\section{The effects of suppression of Ugcg gene expression on neural differentiation of $\mathrm{mESCs}$}

To examine the role of the Ugcg in neural differentiation, we induced neural differentiation of the Ugcg-shRNA transfected mESCs. The EBs derived from shRNA-1 transfected mESCs were adhered on poly-D-lysine/laminin-coated tissue culture dishes for early neural differentiation (Figure 5A). RT-PCR analysis revealed that shRNA-1 tranfection resulted in decrease of $m R N A$ expression of the glial marker (GFAP) and the neuronal marker (MAP-2) whereas expression of early neural precursor cell marker gene, nestin, was not affected (Figure 5B).

The effects of suppression of Ugcg gene expression on the differentiation of mESCs into neural cells were also evaluated by immunofluorescence staining for nestin, MAP-2, and GFAP. No significant difference was found in nestin expression between the control mESCs and the transfected mESCs (Figure 5C). However, the expressions of GFAP and MAP-2 were significantly reduced in shRNA-1 transfected mESCs in comparison to that of the control mESCs and mocktransfected mESCs (Figures 5D and 5E).

\section{Discussion}

Recently, numerous studies have reported that glycosphingolipids play a crucial role during the development of neural cells. Our previous work showed that daunorubicin-induced overexpression of ganglioside enhanced neurite formation in mESCs (Lee et al., 2007). However, the study could not completely exclude the possibility of side effects induced by chemicals, and the exact roles of glycosphingolipids in neural differentiation of mESCs still remained to be clarified. Therefore, in this study we specifically inhibited Ugcg gene expression using shRNA transfection to determine if glycosphingolipids are involved in neural differentiation of mESCs. RT-PCR revealed that the shRNA transfection significantly decreased the Ugcg gene expression in mESCs. In addition, the expression of the gangliosides, GM3 and GD3, in undifferentiated mESCs was also decreased by Ugcg-shRNA transfection. These results confirmed that inhibition of Ugcg gene expression decreased the expression of glycosphingolipids in mESCs.

The biological roles of glycosphingolipids in cell culture include modification of cellular growth, proliferation, and differentiation. Although functions of glycosphingolipids might be various depending on the cell type, expression levels of glycosphingolipids, and the cell type-specific MAPK pathway (Kamimura et al., 2005), our current study demonstrated that suppression of Ugcg gene expression in mESCs inhibited cellular proliferation possibly by reduction of MAPK activation. These results are consistent with the previous findings that the depletion of glycosphingolipids inhibited proliferation of P19 cells and mESCs (Liour and Yu, 2002; Heo et al., 2006), MAPK pathway in embryonic neural precursor cells (Yanagisawa et al., 2005). However, we can not exclude the possibility that ceramide accumulation caused by suppression of Ugcg gene expression results in apoptotic cell death and cell cycle arrest during cell growth and differentiation (Jayadev et al., 1995; Hatanaka et al., 1998). It has been reported that accumulated ceramide concentration might affect the amount of diverse sphingolipid metabolites such as sphingomyelin, sphingosine, and sphingosine-1-phosphate affecting cell growth, apoptosis and the ability of neural cells (Hannun and Linardic, 1993; Pettus et al., 2004; Tani et al., 2007).

To determine the neural differentiation capability of the Ugcg-suppressed mESCs, we evaluated the RA-induced neural differentiation of the EBs (Bain et al., 1995; Li et al., 1998). A previous study demonstrated that expression of neural cell markers and glycosphingolipids was closely related to 
A

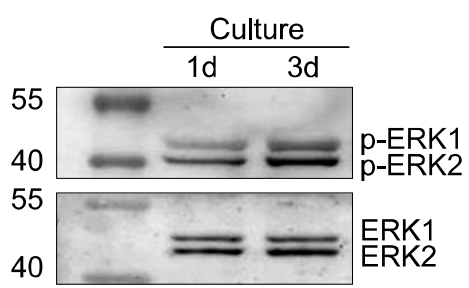

$(\mathrm{kDa})$

C
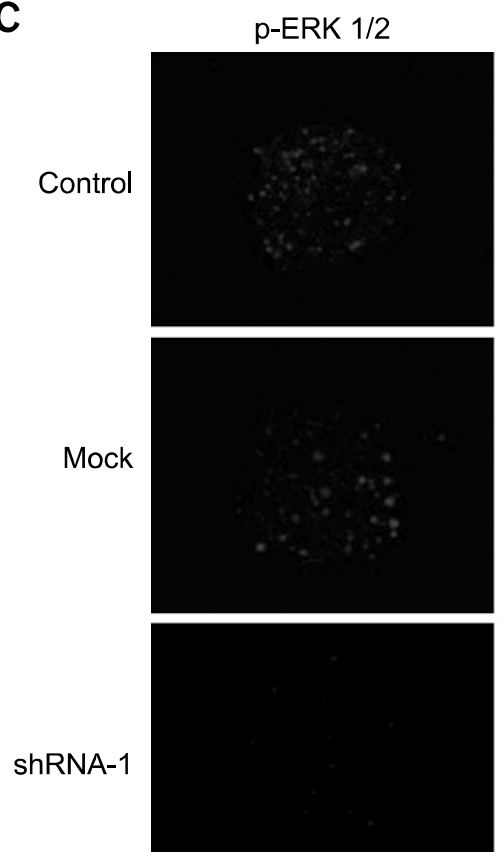

B

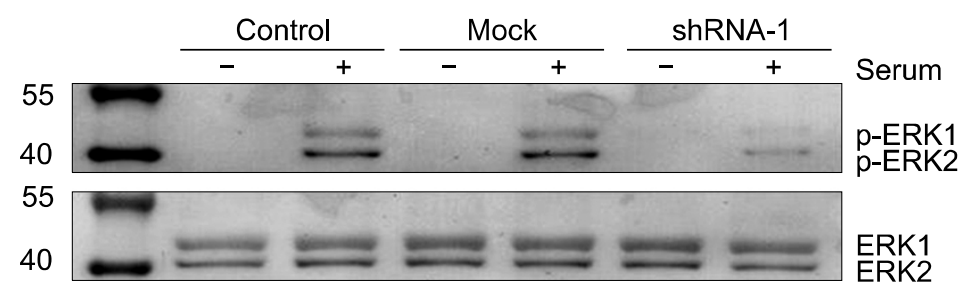

$(\mathrm{kDa})$

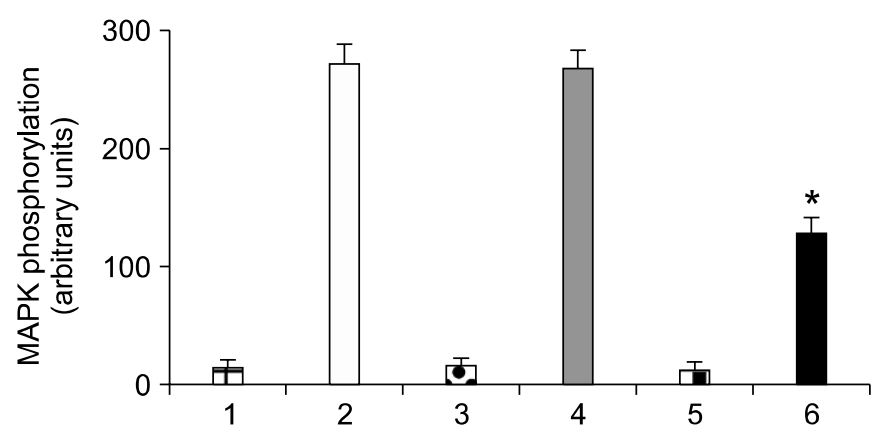

Figure 4. Effect of Ugcg shRNA on MAPK activation of mESCs. (A) MAPK activation of control mESCs was examined by Western blot analysis using anti-phospho-ERK1/2. The results were normalized to ERK1/2 expression. The ERK1/2 in the mESCs was phosphorylated during maintenance under normal culture conditions. 1d, 1day in culture; $3 \mathrm{~d}, 3$ days in culture. (B) Control mESCs, mock-transfected mESCs and shRNA-transfected mESCs were grown for $6 \mathrm{~h}$ in serum-free medium and then stimulated with serum for $10 \mathrm{~min}$. The cells were lysed $10 \mathrm{~min}$ after stimulation and then the cell lysates were examined by Western blot analysis. The results represent the mean \pm SD of the level of phosphorylation observed in three experiments. Lane 1 , control under serum-free condition; lane 2, control under serum condition; lane 3, mock-transfected under serum-free condition; lane 4, mock-transfected under serum condition; lane 5, shRNA-transfected under serum-free condition; lane 6, shRNA-transfected under serum condition. ${ }^{*} P<0.05$ (C) phosphorylation of the ERK1/2 was examined by immunofluorescence staining.

the formation of neurites in cultured neuroblastroma cells (Ferreira et al., 1990). Consistently, we found that expression of GFAP and MAP-2 was barely detectable in Ugcg-suppressed mESCs whereas the level of nestin during neural differentiation was not changed in shRNA-transfected mESCs. Our results suggest that although glycosphingolipids may not play critical functional roles in 
A

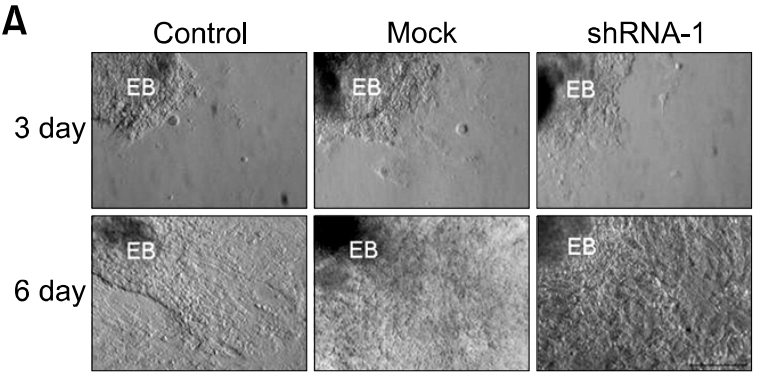

C
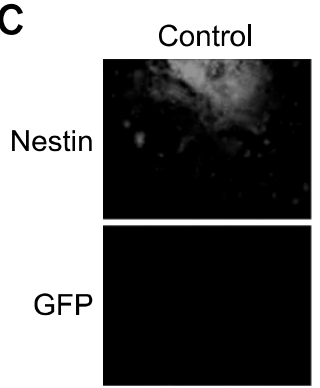

Merge
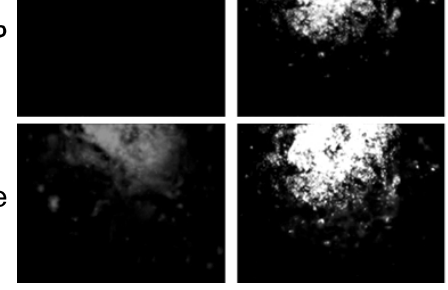

E
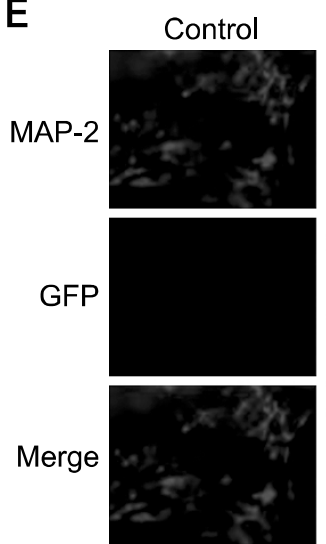

Mock
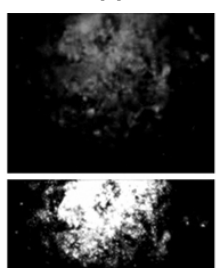

Mock
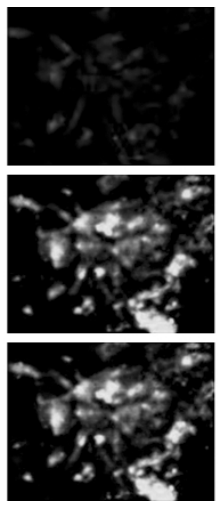

shRNA-1
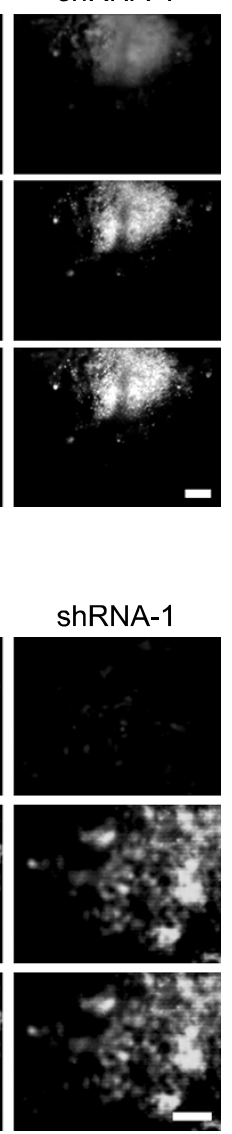

shRNA-1
B

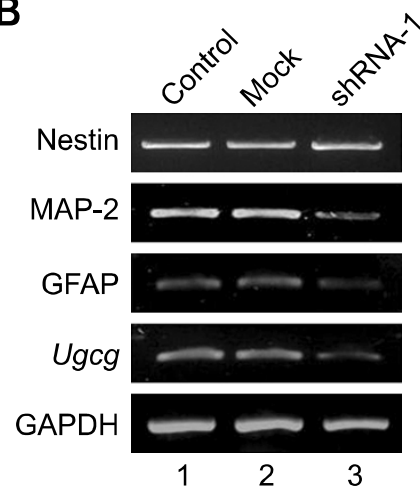

D
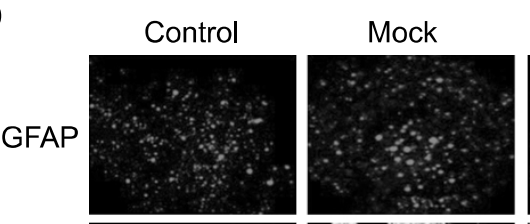

shRNA-1

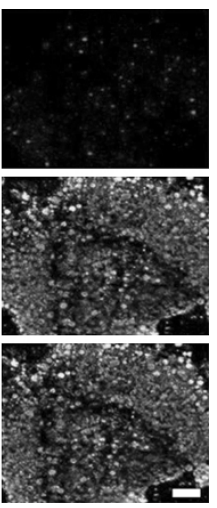

Figure 5. Influence of the suppression of Ugcg gene expression on neural differentiation of mESCs. EBs derived from stably transfected mESCs were dissociated and replated onto a gelatin-coated 6 well dish. EBs were incubated for 4 days to induce early neural differentiation. (A) Morphology of neural cells derived from mESCs induced by RA. 3 days, 3 days in culture; 6 days, 6 days in culture. The scale bar represents $250 \mu \mathrm{m}$. (B) RT-PCR analysis of the various genes (nestin, MAP-2, GFAP, Ugcg) mRNA level of neural cells derived from control, mock-transfected and shRNA-transfected mESCs. RT-PCR using primer sets for the appropriate genes. (C-E) show an immunofluorescence analysis conducted to confirm the expression of nestin, GFAP and MAP-2, respectively. The cells were analyzed using a confocal fluorescence microscope (Model FV300, Olympus Co., Tokyo, Japan). The scale bar represents $100 \mu \mathrm{m}$.

the maintenance of pluripotency in mESCs, which has also been shown in hESCs (Brimble et al., 2007), they have significant roles in neural differentiation process, especially neural maturation process related to GFAP and MAP-2 expression. The current notion that glycosphingolipids are involved in later neural maturation process is supported by our previous study that overexpression of glycosphingolipids can facilitate neurite formation, which is one of neural maturation processes (Lee et al., 2007).

In this study, we found that that suppression of 
Ugcg gene expression inhibited ESC proliferation, possibly associated with reduction of MAPK activation. It is unknown whether reduction of ESC proliferation is exclusively due to the suppression of the ERK $1 / 2$ activity, or possibly also due to alteration in expression of sphingolipid metabolites by inhibition of Ugcg gene expression. Therefore, further studies are still need to check whether inhibition of Ugcg gene expression alters generation of sphingolipid metabolites, which may affect proliferation of mESCs. Our results demonstrated that inhibition of glycosphingolipid expression by downregulation of Ugcg gene expression did not affect differentiation of mESCs to nestin expressing neural precursor cells, suggesting that glycosphingolipids may not have a critical role in early neural commitment of mESCs (Kawai et al., 1998; Lee et al., 2007). Most significant effect of inhibition of glycosphingolipid expression was found in reduction of neural cell marker genes (GFAP and MAP-2). It suggests that the major role of glycosphingolipids is in differentiation of neural precursor cells into neuronal and glial cells. To understand the underlying mechanism by which glycosphingolipids control neural differentiation process in mESCs, future study is required to check whether there is direct regulatory pathway for glycosphingolipids to control expression of later neural cell specific genes.

\section{Methods}

\section{mESCs culture and neural differentiation}

mESC line $\mathrm{J} 1$ was maintained in an undifferentiated status on mouse embryo fibroblast cells in growth medium containing Dulbecco's modified Eagle medium (DMEM) (Gibco, Rockville, MD) supplemented with non-essential amino acids, $2 \mathrm{mM}$ L-glutamine, $0.1 \mathrm{mM} \beta$-mercaptoethanol, leukemia inhibitory factor $(1,000 \mathrm{unit} / \mathrm{ml})$ (Chemicon, Temecula, CA), penicillin (100 unit/ml), streptomycin $(100 \mu \mathrm{g} / \mathrm{ml})$, and $15 \%(\mathrm{v} / \mathrm{v})$ fetal bovine serum (FBS) (Hyclone, Logan, UT), at $37^{\circ} \mathrm{C}$ in $5 \% \mathrm{CO}_{2}$. The feeder cells were treated with mitomycin solution $(10 \mu \mathrm{g} / \mathrm{ml})$ for $3 \mathrm{~h}$ (Lee et al., 2007). In vitro early neural differentiation was induced in mESCs using the 4-/4+ protocol (Bain et al., 1995). The mESCs were grown to form unattached embryoid bodies (EBs) (4-) for 4 days in cell differentiation medium containing DMEM (Gibco, Rockville, MD) supplemented with nonessential amino acids, $2 \mathrm{mM}$ L-glutamine, penicillin $(100 \mathrm{unit} / \mathrm{ml})$, streptomycin $(100 \mu \mathrm{g} / \mathrm{ml})$, and $10 \%$ heat-inactivated FBS (Hyclone, Logan, UT). The EBs (4-) were cultured to generate EB $(4+)$ in the presence of all-trans retinoic acid $(2 \mu \mathrm{M})$ for 4 days. The EBs $(4+)$ were rinsed off and transferred to poly-D-lysine/laminin-coated tissue culture dishes and then incubated overnight in knockout-DMEM (Gibco) with $10 \%$ heat-inactivated FBS to attach the cells to the dish. The EBs (4+) were then rinsed off and grown to induce early neural differentiation in DMEM/F12 medium (Gibco).

\section{Plasmid construction and cell transfection}

A shRNA kit was used to construct the mouse Ugcg gene (UDP-glucose ceramide glucosyltransferase; GenBank. Accession No. NM_011673) (SuperArray Bioscience Corp., Frederick, MD)-targeted shRNAs expression vectors. Ugcg-shRNAs (cloned-1, cloned-2, cloned-3 and cloned-4) were cloned into the linearlized pGeneClip ${ }^{\mathrm{TM}}$ hMGFP vector (Promega Corp., Madison, WI) (Figure 1B). The most effective shRNA clone was selected based on transcriptional repression of the target gene. For stable transfection with targeting vectors, mESCs $\left(1 \times 10^{5}\right.$ cells/well $)$ were plated in 6-well gelatin-coated plates without mouse embryonic fibroblast feeder cells and then transfected with either circular targeting vectors or linearized targeting vectors using Lipofectamine 2000 according to the manufacturer's instructions (Invitrogen, Carlsbad, CA). After 24 $\mathrm{h}$ of incubation at $37^{\circ} \mathrm{C}$ in $5 \% \mathrm{CO}_{2}$, the medium was changed to the standard medium described above. Individual clones with GFP fluorescence were identified, picked, and expanded.

\section{Cell proliferation assay}

Cells were seeded at a density of $4 \times 10^{4}$ cells/well in 24 -well plates and then transfected for $24 \mathrm{~h}$ at $37^{\circ} \mathrm{C}$. Forty eight hours after transfection, cell proliferation was determined by MTT assay. Each well was incubated in MTT [3-(4,5-Dimethylthiazol-2-yl)-2,5-diphenyl-tetrazolium bromide] (Sigma) solution for $4 \mathrm{~h}$, and the absorbance was then determined at $490 \mathrm{~nm}$ using a spectrophotometer. The experiments were repeated four times, and the cell proliferation data were analyzed using analysis of variance (ANOVA) followed by Tukey's multiple comparison test to determine if significant differences existed between groups $(P<0.05)$.

\section{Ganglioside extraction}

Gangliosides were extracted and purified using previously described methods (Ladisch and Gillard, 1985). Briefly, the cells were homogenized and the total lipids were then extracted in distilled water at $4^{\circ} \mathrm{C}$. Next, the extracted total lipid in chloroform/methanol $(1: 1, \mathrm{v} / \mathrm{v})$ was evaporated under $\mathrm{N}_{2}$ gas and then dissolved in chloroform/methanol/ $\mathrm{H}_{2} \mathrm{O}(15: 30: 4, \mathrm{v} / \mathrm{v} / \mathrm{v})$. The dissolved total lipid sample was then applied to a diethylaminoethyl sephadex A25 column (Sigma) and washed with chloroform $/$ methanol $/ \mathrm{H}_{2} \mathrm{O}$ $(15: 30: 4, v / v / v)$ to remove the neural lipids. The acidic lipids were then eluted by adding chloroform/methanol/sodium acetate $(15: 30: 4, \mathrm{v} / \mathrm{v} / \mathrm{v})$ to the column. Next, the eluted samples were dried at $30^{\circ} \mathrm{C}$ under $\mathrm{N}_{2}$ for $5 \mathrm{~h}$, dissolved in chloroform/methanol $(1: 1, \mathrm{v} / \mathrm{v})$, and alkalized in $12 \mathrm{~N}$ ammonium hydroxide solution. The acidic lipid samples dissolved in chloroform/methanol $(1: 1, \mathrm{v} / \mathrm{v})$ were then applied to a Sep-Pak C18 cartridge column, and then washed with $\mathrm{H}_{2} \mathrm{O}$ to remove the non-hydrophobic lipids. The gangliosides were then eluted by adding methanol, after which they were dried at $30^{\circ} \mathrm{C}$ under $\mathrm{N}_{2}$ for $3 \mathrm{~h}$ and 
then stored at $-80^{\circ} \mathrm{C}$

\section{High-performance thin-layer chromatography (HPTLC)}

HPTLC analysis of the gangliosides was performed using $10 \times 10$ or $10 \times 20 \mathrm{~cm}$ thin-layer chromatography 5651 plates (Merck, Darmstadt, Germany). The purified gangliosides (1 mg protein/lane) were loaded onto thin-layer chromatography 5651 plates (Ladisch and Gillard, 1985), which were then developed in chloroform $/$ methanol $/ 0.25 \%$ $\mathrm{CaCl}_{2} \cdot \mathrm{H}_{2} \mathrm{O}(50: 40: 10, \mathrm{v} / \mathrm{v} / \mathrm{v})$. The gangliosides were stained with resorcinol- $\mathrm{HCl}$ reagent. Bovine brain gangliosides were used as a standard to categorize the individual ganglioside species.

\section{Immunofluorescence analysis}

Cells were fixed with $4 \%$ paraformaldehyde in phosphate-buffered saline (PBS) for 15 mins at room temperature and permeabilized with $0.25 \%$ Triton $X-100$ for 10 mins at $37^{\circ} \mathrm{C}$. The cells were then blocked with PBS containing $4 \%$ BSA for 20 mins followed by incubation for $16 \mathrm{~h}$ at $4^{\circ} \mathrm{C}$ with primary antibodies specific to neural cell markers [nestin (Chemicon International Inc., Temecula, CA), GFAP (glial fibrillary acidic protein) (Santa Cruz Biotechnology Inc., Santa Cruz, CA) and MAP-2 (microtubule-associated protein 2) (Sigma)], stage specific embryonic antigen-1 (SSEA-1) (Chemicon), phospho-extracellular signal-regulated kinase $1 / 2$ (ERK1/2) (Promega) and gangliosides (GM3, GD3) (Seikagaku Corp., Tokyo, Japan) in PBS containing 1\% BSA. The cells were washed with PBS containing 1\% BSA followed by incubation with secondary antibodies conjugated to fluorescent markers [goat anti-rabbit IgG-Alexa fluor 350, goat anti-rabbit IgG-Alexa fluor 488, goat anti-mouse IgM-Alexa fluor 350 (Molecular Probes, Carlsbad, CA), and goat anti-mouse IgG-TRITC (Sigma)] at 1:500 dilution. To detect alkaline phosphatase (AP), mESC colonies were fixed in $4 \%$ formaldehyde for $15 \mathrm{~min}$ and stained with Fast red TR/naphthol AS-MX for 15 min (Sigma). After washed with PBS, the cells were analyzed using a fluorescence microscope (Carl Zeiss Inc., Germany) and a confocal fluorescence microscope (Model FV300, Olympus Co., Japan).

\section{Western blot analysis}

Cells were homogenized with lysis buffer $[50 \mathrm{mM}$ Tris- $\mathrm{HCl}$ (pH 8.0), $150 \mathrm{mM} \mathrm{NaCl}, 0.02 \%$ sodium azide, $0.1 \% \mathrm{SDS}, 1$ mM PMSF, $1 \mathrm{mM} \mathrm{Na}_{3} \mathrm{VO}_{4}, 1 \mathrm{mM} \mathrm{NaF}, 1 \%$ Triton X-100, 0.5 $\mathrm{mM}$ EDTA, and $0.5 \%$ sodium deoxycholate] and then centrifuged at $15,000 \mathrm{~g}$ for $30 \mathrm{~min}$. The protein concentration was then measured according to the Bradford method. Equal amounts of proteins $(40 \mu \mathrm{g})$ were separated on a SDS/7\%-polyacrylamide gel and then transferred to a nitrocellulose membrane (Amersham Biosciences, Piscataway, NJ). The blots were blocked for $2 \mathrm{~h}$ with $5 \%(\mathrm{w} / \mathrm{v})$ non-fat dried milk in Tris-buffered saline (TBS) [10 mM Tris? $\mathrm{HCl}(\mathrm{pH} 8.0)$ and $150 \mathrm{mM} \mathrm{NaCl}, 0.05 \%$ Tween-20]. The membrane was incubated for $16 \mathrm{~h}$ at $4^{\circ} \mathrm{C}$ with rabbit IgGs specific to ERK1/2, phospho-ERK1/2 (Promega), respectively. The blot was incubated with anti-rabbit alkaline phosphatase-conjugated secondary antibody (Sigma). After incubation in BCIT/NBT (Zymed, San Francisco, CA), the membranes were scanned with Microtek ScanMaker 5 and quantified by densitometry analysis (Beta 4.0.3 of Scion Image, Frederick, MD).

\section{RNA extraction and reverse transcription-PCR mRNA analysis}

Total RNA from the mESCs was isolated using a TRI reagent (Molecular Research Center, Inc., Cincinnati, $\mathrm{OH}$ ). RT-PCR was conducted to exam expression of the Oct3/4, Sox2, nestin, MAP-2, GFAP and Ugcg using the one-step RT-PCR kit (Promega) with a Takara PCR Thermal Cycler DICE Gradient (Takara, Shiga, Japan) under the following conditions: $94^{\circ} \mathrm{C}$ for 5 mins, followed by 30 cycles of $94^{\circ} \mathrm{C}$ for $30 \mathrm{sec}, 60^{\circ} \mathrm{C}$ for $30 \mathrm{sec}$, and $72^{\circ} \mathrm{C}$ for $30 \mathrm{sec}$, with a final extension at $72^{\circ} \mathrm{C}$ for 7 mins. PCR was conducted using the following primer sets: forward (5'-GGCGTTCTCTTTGGAAAGGTGTTC-3') and reverse (5'-CTCGAACCACATCCTTCTCT-3') for Oct $3 / 4$; forward (5'-GGCAGCTACAGCATGATGCAGGAGC-3') and reverse (5'-CTGGTCATGGAGTTGTACTGCAGG-3') for Sox2; forward (5'-AGACAGTGAGGCAGATGAGT-3') and reverse (5'-ATGAGAGGTCAGAGTCATGG-3') for nestin; forward (5'-CTGGTGCTTTTAAACAGGCG-3') and reverse (5'-TTGCAGTTGATCCAGGGGTA-3') for MAP-2; forward (5'-GCTCAATGACCGCTTTGCTA-3') and reverse (5'-TGCAAAGTTGTCCCTCTCCA-3') for GFAP; forward (5'-CCCAAATGTCGATGC-3') and reverse (51'-AAGGTGGCAGCAAAG-3') for Ugcg; and forward (5'-TTCACCACCATGGAGAAGGCT-3') and reverse (5'-GACAACCTGGTCCTCAGTGTA-3') for GAPDH.

\section{Supplemental data}

Supplemental Data include two figures and can be found with this article online at http://e-emm.or.kr/ article/article files/SP-41-12-02.pdf.

\section{Acknowledgements}

We thank Dr. Naoyuki Taniguchi (Department of Biochemistry, Osaka University Graduate School of Medicine, Osaka, Japan) for numerous suggestions and comments regarding the subjects discussed in this study. This work was supported by Wonkwang University in 2008.

\section{References}

Allende ML, Proia RL. Lubricating cell signaling pathways with gangliosides. Curr Opin Struct Biol 2002;12:587-92

Bain G, Kitchens D, Yao M, Huettner JE, Gottlieb DI. Embryonic stem cells express neuronal properties in vitro. Dev Biol 1995;168:342-57

Brimble SN, Sherrer ES, Uhl EW, Wang E, Kelly S, Merrill Jr AH, Robins AJ, Schulz TC. The cell surface glycosphingolipids SSEA-3 and SSEA-4 are not essential for 


\section{human ESC pluripotency. Stem Cells 2007;25:54-62}

Diaz-Font A, Chabás A, Grinberg D, Vilageliu L. RNAimediated inhibition of the glucosylceramide synthase (GCS) gene: A preliminary study towards a therapeutic strategy for Gaucher disease and other glycosphingolipid storage diseases. Blood Cells Mol Dis 2006;37:197-203

Draper JS, Pigott C, Thomson JA, Andrews PW. Surface antigens of human embryonic stem cells: changes upon differentiation in culture. J Anat 2002;200:249-58

Ferreira A, Busciglio J, Landa C, Caceres A. Ganglioside-enhanced neurite growth: evidence for a selective induction of high-molecular-weight MAP-2. J Neurosci 1990;10:293-302

Fukumoto S, Mutoh T, Hasegawa T, Miyazaki H, Okada M, Goto G, Furukawa K, Urano T. GD3 synthase gene expression in $\mathrm{PC} 12$ cells results in the continuous activation of TrkA and ERK $1 / 2$ and enhanced proliferation. J Biol Chem 2000;275:5832-8

Hannun YA, Linardic CM. Sphingolipid breakdown products: anti-proliferative and tumor-suppressor lipids. Biochim Biophys Acta 1993;1154:223-36

Hatanaka Y, Fujii J, Fukutomi T, Watanabe T, Che W, Sanada $\mathrm{Y}$, Igarashi $\mathrm{Y}$, Taniguchi N. Reactive oxygen species enhances the induction of inducible nitric oxide synthase by sphingomyelinase in RAW264.7 cells. Biochim Biophys Acta 1998;1393:203-10

Heo JS, Lee YJ, Han HJ. EGF stimulates proliferation of mouse embryonic stem cells: involvement of $\mathrm{Ca} 2+$ influx and p44/42 MAPKs. Am J Physiol Cell Physiol 2006;290: C123-33

Jayadev S, Liu B, Bielawska AE, Lee JY, Nazaire F, Pushkareva MY, Obeid LM, Hannun YA. Role for ceramide in cell cycle arrest. J Biol Chem 1995;270:2047-52

Kamimura Y, Furukawa K, Kittaka D, Nishio M, Hamamura K, Fukumoto S, Furukawa K. Differential enhancing effects of alpha2,8-sialyltransferase on the cell proliferation and mobility. Int J Oncol 2005;26:337-44

Kawai H, Sango K, Mullin KA, Proia RL. Embryonic stem cells with a disrupted GD3 synthase gene undergo neuronal differentiation in the absence of b-series gangliosides. J Biol Chem 1998;273:19634-8

Kawai H, Allende ML, Wada R, Kono M, Sango K, Deng C, Miyakawa T, Crawley JN, Werth N, Bierfreund U, Sandhoff $\mathrm{K}$, Proia RL. Mice expressing only monosialoganglioside GM3 exhibit lethal audiogenic seizures. J Biol Chem 2001;276:6885-8

Kimber SJ, Brown DG, Pahlsson P, Nilsson B. Carbohydrate antigen expression in murine embryonic stem cells and embryos. II. Sialylated antigens and glycolipid analysis. Histochem J 1993;25:628-41

Kimura M, Hidari KI, Suzuki T, Miyamoto D, Suzuki $Y$ Engagement of endogenous ganglioside GM1a induces tyrosine phosphorylation involved in neuron-like differentiation of PC12 cells. Glycobiology 2001;11:335-43

Kiura K, Watarai S, Ueoka H, Tabata M, Gemba K, Aoe K,
Yamane H, Yasuda T, Harada M. An alteration of ganglioside composition in cisplatin-resistant lung cancer cell line. Anticancer Res 1998;18:2957-60

Ladisch S, Gillard B. A solvent partition method for microscale ganglioside purification. Anal Biochem 1985; 146:220-31

Lee DH, Koo DB, Ko K, Ko K, Kim SM, Jung JU, Ryu JS, Jin JW, Yang HJ, Do SI, Jung KY, Choo YK. Effects of daunorubicin on ganglioside expression and neuronal differentiation of mouse embryonic stem cells. Biochem Biophys Res Commun 2007;362:313-8

Li M, Pevny L, Lovell-Badge R, Smith A. Generation of purified neural precursors from embryonic stem cells by lineage selection. Curr Biol 1998;27:971-4

Liour SS, Kapitonov D, Yu RK. Expression of gangliosides in neuronal development of P19 embryonal carcinoma stem cells. J Neurosci Res 2000;62:363-73

Liour SS, Yu RK. Differential effects of three inhibitors of glycosphingolipid biosynthesis on neuronal differentiation of embryonal carcinoma stem cells. Neurochem Res 2002; $27: 1507-12$

Liu YY, Han TY, Yu JY, Bitterman A, Le A, Giuliano AE, Cabot $M C$. Oligonucleotides blocking glucosylceramide synthase expression selectively reverse drug resistance in cancer cells. J Lipid Res 2004;45:933-40

Maupas-Schwalm F, Augé N, Robinet C, Cambus JP, Parsons SJ, Salvayre R, Nègre-Salvayre A. The sphingomyelin/ceramide pathway is involved in ERK1/2 phosphorylation, cell proliferation, and UPAR overexpression induced by tissue-type plasminogen activator. FASEB J 2004;18:1398-400

Murozuka Y, Watanabe N, Hatanaka K, Hakomori SI. Lyso-GM3, its dimer, and multimer: their synthesis, and their effect on epidermal growth factor-induced receptor tyrosine kinase. Glycoconj J 2007;24:551-63

Pettus BJ, Chalfant CE, Hannun YA. Sphingolipids in inflammation: roles and implications. Curr Mol Med 2004; 4:405-18

Prinetti A, Millimaggi D, D'Ascenzo S, Clarkson M, Bettiga A Chigorno V, Sonnino S, Pavan A, Dolo V. Lack of ceramide generation and altered sphingolipid composition are associated with drug resistance in human ovarian carcinoma cells. Biochem J 2006;395:311-8

Proia RL. Glycosphingolipid functions: insights from engineered mouse models. Philos Trans R Soc Lond B Biol Sci 2003;358:879-83

Stoica BA, Movsesyan VA, Knoblach SM, Faden AI. Ceramide induces neuronal apoptosis through mitogenactivated protein kinases and causes release of multiple mitochondrial proteins. Mol Cell Neurosci 2005;29:355-71

Tani M, Ito M, Igarashi Y. Ceramide/sphingosine/sphingosine 1-phosphate metabolism on the cell surface and in the extracellular space. Cell Signal 2007;19:229-37

Van Echten G, Sandhoff K. Ganglioside metabolism. Enzymology, Topology, and regulation. J Biol Chem 1993 268:5341-4 
Van Meer G. Transport and sorting of membrane lipids. Curr Opin Cell Biol 1993;5:661-73

Yamashita T, Wada R, Sasaki T, Deng C, Bierfreund U, Sandhoff K, Proia RL. A vital role for glycosphingolipid synthesis during development and differentiation. Proc Natl Acad Sci USA 1999;96:9142-7
Yanagisawa M, Nakamura K, Taga T. Glycosphingolipid synthesis inhibitor represses cytokine-induced activation of the Ras-MAPK pathway in embryonic neural precursor cells. J Biochem 2005;138:285-91

Yu RK. Development regulation of ganglioside metabolism. Prog Brain Res 1994;101:31-44 